\title{
Glioblastoma multiforme has many faces
}

\section{P van Rensburg, $M B$ ChB}

Department of Radiology, Steve Biko Academic Hospital and University of Pretoria

\section{M van Rensburg, FRCR, FRANZCR}

Neuroradiologist in private practice, Life Wilgers Hospital, Pretoria

Corresponding author: P van Rensburg (pjvanr@gmail.com)

Glioblastoma multiforme (GBM) is a class of devastating, highly aggressive central nervous system tumours. While the classical appearance is easily recognisable, several variations occur. We present 6 cases of confirmed GBM that illustrate the unusual findings in histological subtypes, early presentation and spread on MRI imaging.

S Afr J Rad 2012;16(3):94-99. DOI:10.7196/SAJR.678

Gliomas are central nervous system (CNS) neoplasms with specific histological characteristics that comprise almost $60 \%$ of all CNS malignancies. ${ }^{1}$ They are subdivided into astrocytomas and oligodendrogliomas. ${ }^{1}$ The most common form of gliomas are the astrocytomas, and the most aggressive type of astrocytoma is glioblastoma multiforme (GBM). ${ }^{1}$ Surgery, chemotherapy and radiation therapy achieve little success in the management of glioblastoma. Despite intensive research into treatment options, the median life expectancy for patients with glioblastoma remains unchanged at 12

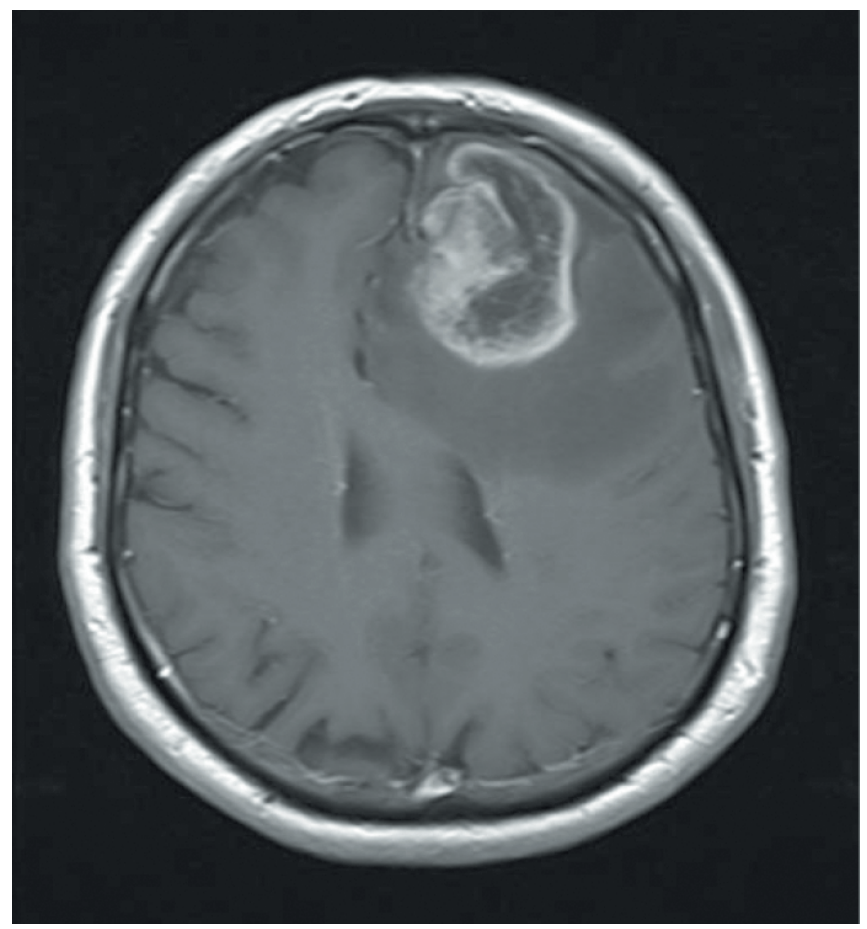

Fig. 1a. T1 axial post-contrast view shows marked peripheral contrast enhancement and central breakdown. months. Only $5 \%$ of patients will be alive 5 years after diagnosis.

The behaviour of malignant glioma is unusual. Systemic metastases occur but are rare, and most tumours recur within $2 \mathrm{~cm}$ of their original tumour margin. They are so aggressive and invasive that they have been referred to as a 'whole CNS' disease. ${ }^{2}$

Glioblastoma multiforme demonstrates a high degree of heterogeneity in both its pathological and radiological appearance. ${ }^{3}$ We present a series of 5 patients with histologically proven glioblastoma multiforme with atypical MRI findings.

\section{Case 1: Gliosarcoma}

A 64-year-old man was initially admitted for pneumonia. On further inquiry, relatives reported that he had suffered from mild confusion, forgetfulness and inappropriate behaviour for a few weeks prior to admission. The clinical examination was unremarkable. MRI demonstrated a left frontal mass with surrounding oedema and pressure effects including midline shift. The mass had low to intermediate signal on T1WI and high signal on T2 and FLAIR. Peripheral enhancement and central necrosis were noted (Figs 1a, b).The mass was resected and, after histological examination, found to be a gliosarcoma.

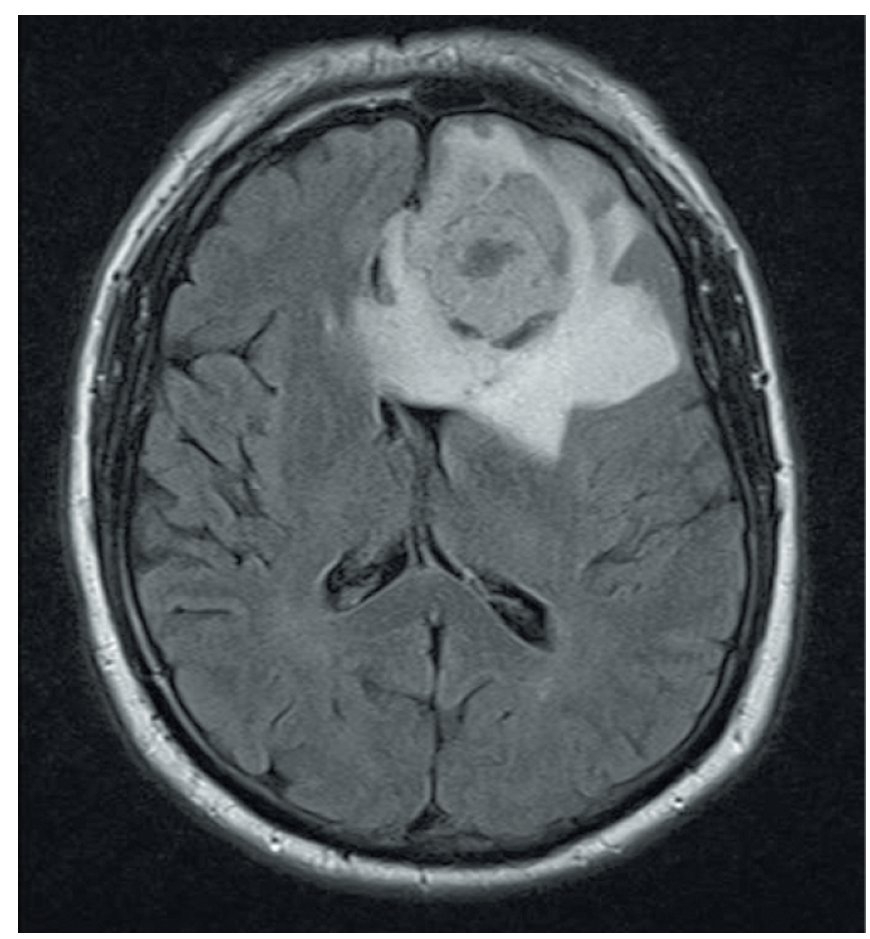

Fig. 1b. FLAIR axial view shows surrounding oedema and associated pressure effects. 


\section{PICTORIAL REVIEW}

\section{Case 2: Meningeal involvement}

A 31-year-old man presented with seizures and subsequent diplopia. Initial imaging revealed a space-occupying, non-enhancing lesion in the optic chiasm. An excision biopsy showed a grade II astrocytoma. Subsequent radiotherapy localised to the lesion and base of the brain was completed. At the 18-month follow-up, he complained of recurrent headaches, and was found to have dural thickening on imaging. A biopsy of the dura over the vertex was non-contributory. A ventriculoperitoneal shunt for hydrocephalus was placed following the negative biopsy, and repeat biopsy at this time was still inconclusive.

MRI following clinical deterioration 6 months after the shunt placement showed marked enhancement of the meninges, especially of the pia mater, extending around the midbrain, into the cerebral gyri and cerebellar folia (Fig. 2). A biopsy was then taken from the meninges in the left sylvian fissure, which confirmed GBM.

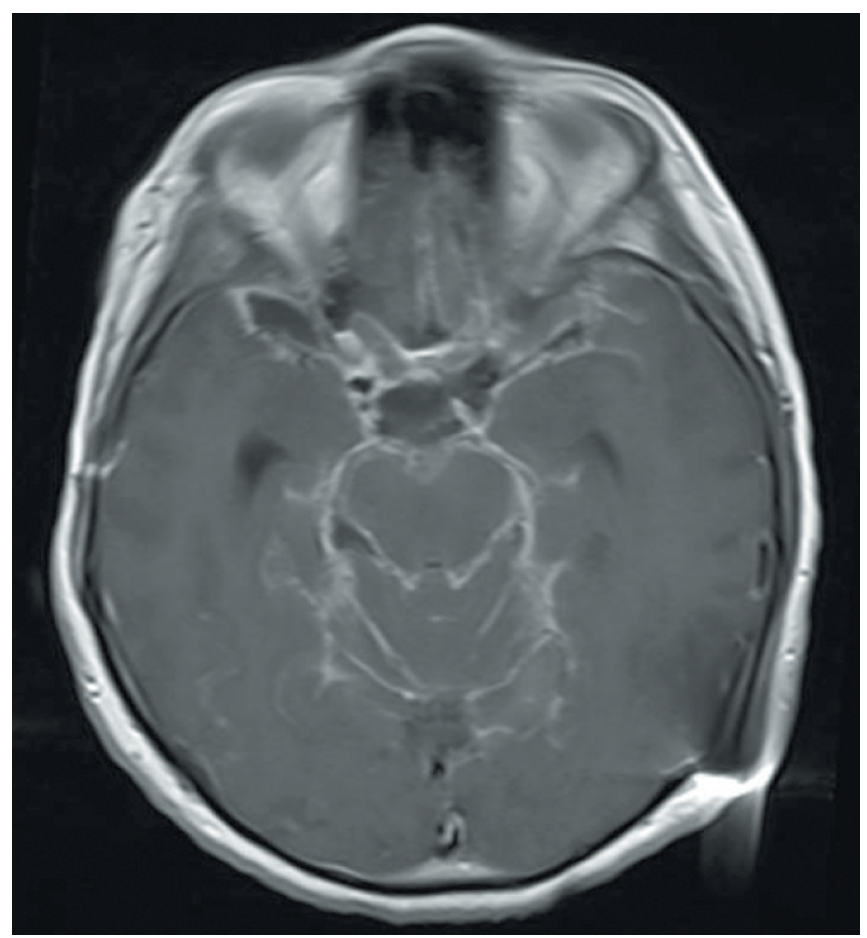

Fig. 2. T1 axial post-contrast images show marked meningeal enhancement extending into the cerebellar folia, around the midbrain and along the left sylvian fissure.

\section{Case 3: Early-stage glioblastoma multiforme}

A 62-year-old man presented with generalised tonic-clonic seizures. The EEG and MRI at this time were reported as normal. He was treated with anticonvulsants and had no further seizures. Five months later, he had an episode of confusion with receptive and expressive aphasia, lasting a few minutes, but no associated seizure. He also complained of feeling blunted, with mild memory impairment, but ascribed this to the medication.No imaging was performed. A further 3 months later, he again experienced expressive aphasia and memory loss. At this stage, a second MRI was performed. A large, heterogenous, left temporal rimenhancing lesion was seen on the follow-up images (Fig. 3a).

Review of the initial non-contrast MRI revealed 2 high-signal foci in the left superior temporal gyrus on the T2 images, confirmed on the coronal FLAIR (Fig. 3b). The lesion was found to be a GBM on histological examination.

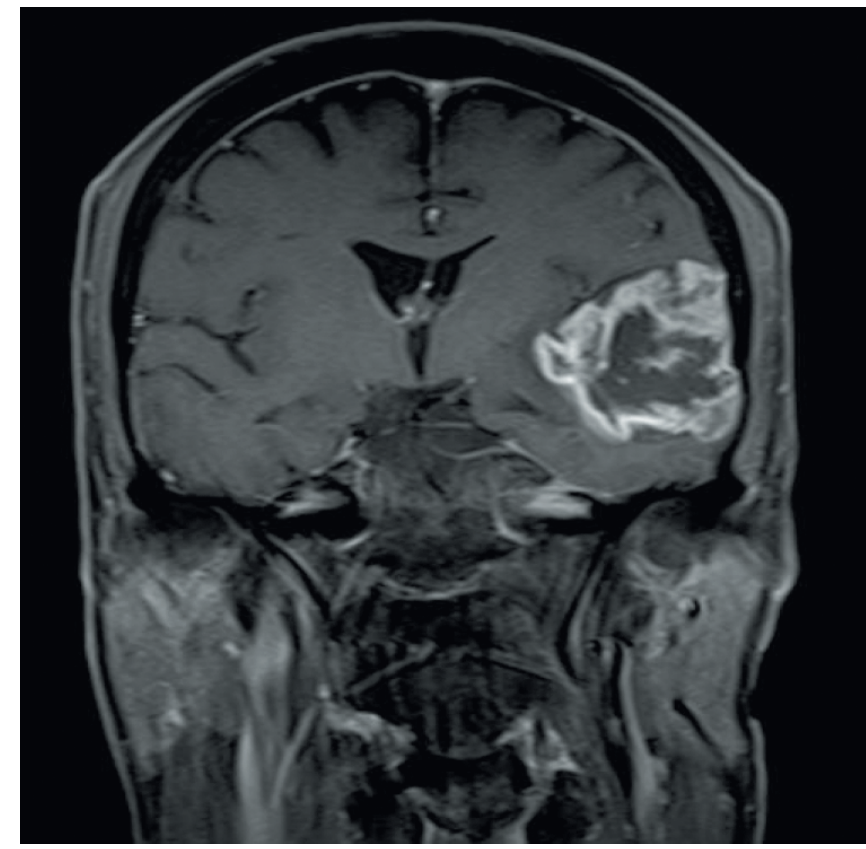

Fig. 3a. T1 coronal post-contrast view shows a large, centrally necrotic, peripherally enhancing tumour.

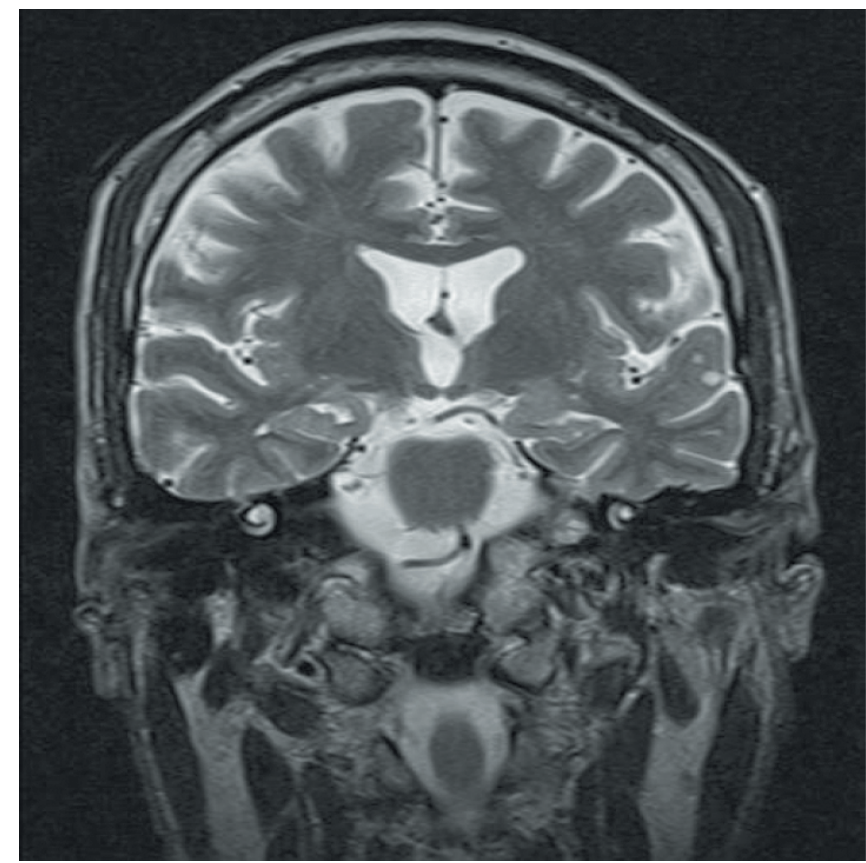

Fig. 3b. T2 coronal view shows small hyperintense foci in the left superior temporal gyrus.

\section{Case 4: Early-stage glioblastoma multiforme}

A 66-year-old woman presented with status epilepticus. The initial MRI (Fig. 4a) showed 2 adjacent areas in the left frontal lobe with high signal on T2WI and FLAIR. These showed high signal on diffusion weighted imaging (DWI) and low apparent diffusion coefficient (ADC) values, with subtle enhancement following contrast administration. Polymerase chain reaction (PCR) testing for Herpes simplex was positive, and the 


\section{PICTORIAL REVIEW}



Fig. 4a. FLAIR axial view shows 2 high-signal foci in the left frontal lobe.

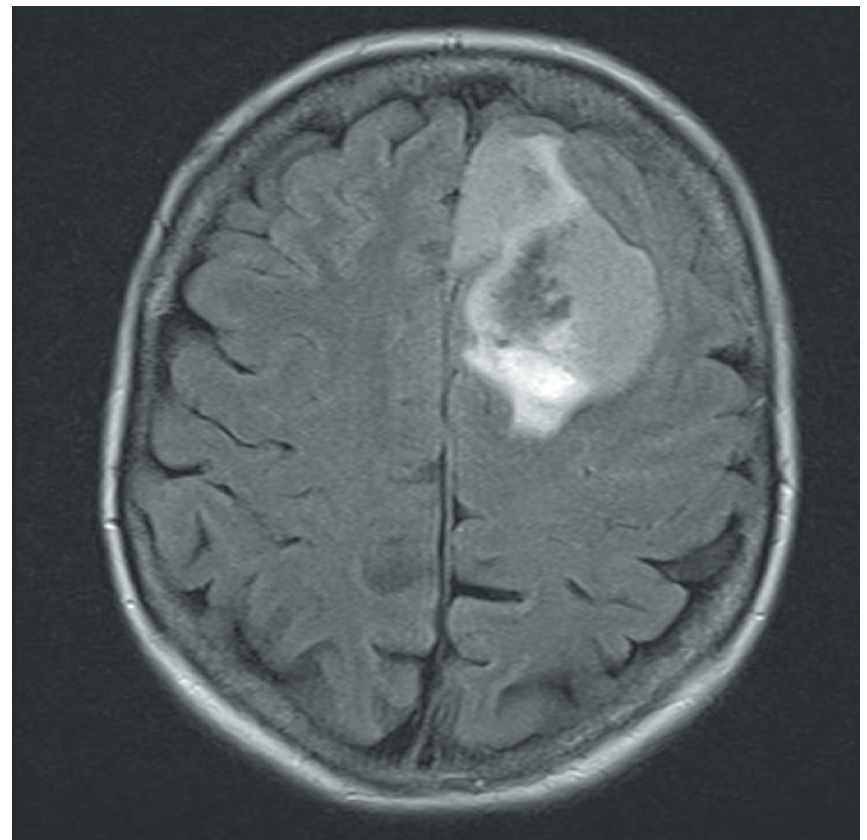

Fig. 4b. Follow-up FLAIR axial view shows a large lesion with mass effect and surrounding oedema.

patient was managed medically. Seven weeks later, she was re-admitted, clinically better and the status had resolved, but there was mild personality change with blunted affect.

Follow-up images (Fig. 4b) demonstrated a large lesion in the left frontal lobe with central breakdown, peripheral enhancement and surrounding oedema. Repeat MRI led to a biopsy, and the lesion was histologically confirmed to be a GBM.

\section{Case 5: Subependymal spread of glioblastoma multiforme}

A 60-year-old man was admitted for left-sided abdominal pain of approximately 3 weeks. A chest radiograph showed nodules in the base of the right lung. During his admission, he had episodes of confusion and experienced nausea and vomiting. MRI a week after admission

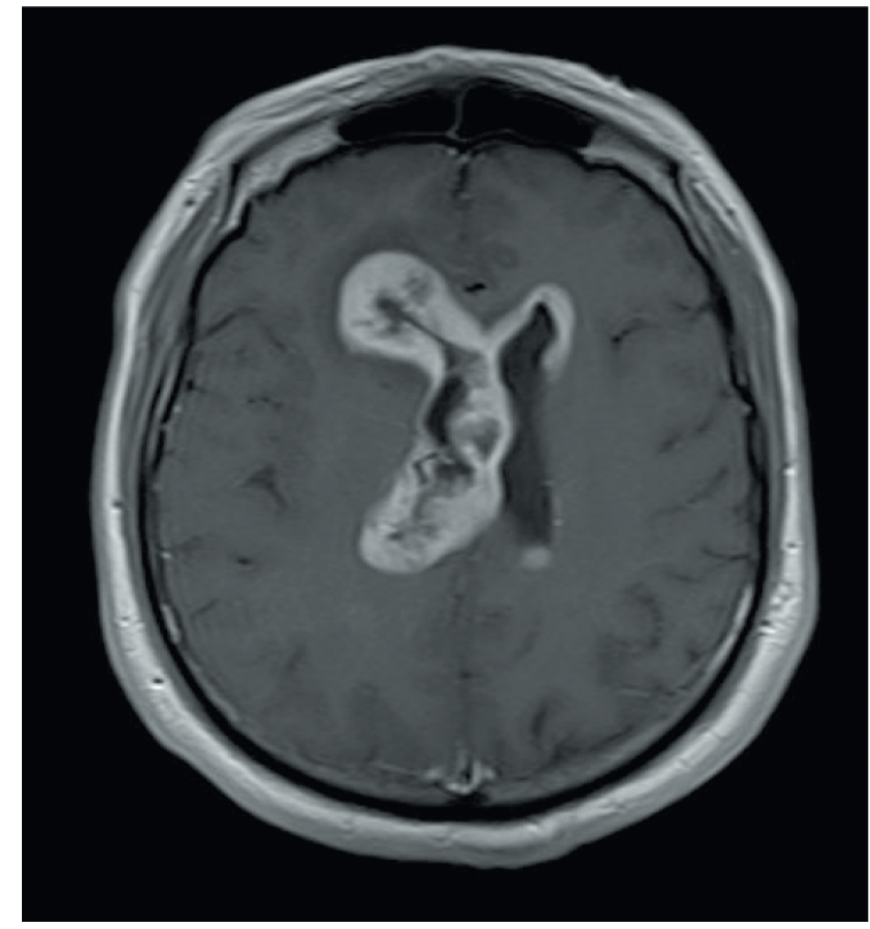

Fig. 5. FLAIR axial post-contrast view shows extensive subependymal infiltration of tumour.

demonstrated subependymal infiltration of tumour in the fourth, third and lateral ventricles. Associated oedema was seen surrounding the frontal horn of the right lateral ventricle. Areas of breakdown are seen within the tumour, notably surrounding the occipital and temporal horns of the right lateral ventricle (Fig. 5). The histology report was that of a GBM.

\section{Discussion}

GBMs normally arise in white matter and are typically solitary, relatively large, irregularly shaped masses with associated necrosis and neovascularity, leading to intra-tumoral arteriovenous shunting. ${ }^{3}$ Infiltration occurs along white matter tracts and perivascular spaces, leading to distant spread despite minimal underlying architectural distortion. $^{3}$

\section{Pathology}

Typical histopathological features include cellular polymorphism, increased mitotic activity, atypical nuclei, vascular thrombosis and microvascular proliferation as well as necrosis. ${ }^{4}$

GBM frequently arises de novo, but may develop from diffuse or anaplastic astrocytomas that undergo de-differentiation. ${ }^{4}$ Glioblastoma is the most common brain tumour, accounting for $12-15 \%$ of intracranial neoplasms and $50-60 \%$ of astrocytic tumours. ${ }^{4}$

\section{Imaging}

MR imaging is crucial in lesion characterisation. Lesions are typically hypo-intense on T1WI and hyperintense on T2WI and FLAIR. Following intravenous contrast administration, findings include nodular and irregular rim enhancement with central hypo-intensity interpreted as necrosis. Ill-defined margins and surrounding oedema are typical. It is important to note that non-enhancing regions may also include 


\section{PICTORIAL REVIEW}

tumour as noted on histological analysis. ${ }^{3}$ Metabolic information may be acquired using MR spectroscopy where there is typically a reduction in NAA (N-acetylaspartate) corresponding to a decreased density of neuronal cells and increased choline as a result of higher cell membrane turnover. $^{3}$

As complete resection is rare, radiotherapy is used to treat residual disease. MRI is useful in monitoring disease progression and in treatment planning. ${ }^{3}$ Computerised tomography, catheter angiography, SPECT and PET play a lesser role in imaging. ${ }^{3}$

Gliosarcoma is a glioblastoma variant with different areas displaying glial and mesenchymal differentiation. ${ }^{4}$ The tumour contains a portion that satisfies the histological criteria for GBM, and a mesenchymal component that may display a variety of morphologies with origins from fibroblastic, cartilaginous, osseous, smooth muscle, striated muscle or adipose cell lineage. There are 2 distinct types of gliosarcoma: one that is similar to a GBM, and one that macroscopically mimics a meningioma that is more amenable to resection and, as such, has a more favourable prognosis. $^{5}$

\section{Multifocal glioblastomas}

The presence of true multiple, independent lesions outside of inherited syndromes remains controversial as, even on careful post mortem studies, the connection between lesions may be difficult to confirm owing to the fact that the infiltrating cells are often small, undifferentiated and polar. ${ }^{4}$

\section{Diffusion abnormalities}

In a subset of patients with glioblastoma, development of a new focus of restricted diffusion during treatment may precede the development of new enhancing tumour. ${ }^{6}$

\section{Spread via cerebrospinal fluid}

Risk factors for cerebrospinal fluid (CSF) dissemination include direct invasion of the ependyma, fissuring of the ependyma secondary to hydrocephalus, fragmentation of tumour in contact with CSF, and surgical intervention. ${ }^{7}$

\section{Conclusion}

While the classic appearance of GBM is one of the more recognisable diagnoses in radiology, it is important to bear in mind that many variations occur. Seemingly innocuous foci of signal abnormality, meningeal pathology and multicentric lesions may represent or progress to GBM.

1. Chengkai D, Holland E. Astrocyte differentiation states and glioma formation. Cancer J 2003;9(2):72-81 2. Market J. Glioblastoma multiforme: Introduction. Cancer I 2003:9(2):71.

3. Nelson SJ, Cha S. Imaging glioblastoma multiforme. Cancer J 2003;9(2):134-145.

4. Louis DN, Ohgaki H, Wiestler OD, et al. Classification of Tumors of the Nervous System. Geneva: World Health Organization, 2007.

5. Han SJ, Yang I, Ahn BJ, et al. Clinical characteristics and outcomes for a modern series of primary gliosarcoma patients. Cancer 2010;116(5):1358-1366.

6. Gupta A, Young RJ, Karimi S, et al. Isolated diffusion restriction precedes the development of enhancing tumor in a subset of patients with glioblastoma. Am J Neuroradiol 2011;32(7):1-6.

7. Alatakis S, Malhamand M, Thien C. Spinal leptomeningeal metastasis from cerebral glioblastoma multiforme presenting with radicular pain. Surg Neurol 2001;56:33-38. 